\title{
Silver Fluoride as a Treatment for Dental Caries
}

\author{
Advances in Dental Research \\ 2018, Vol. 29(I) I35-140 \\ (C) International \& American Associations \\ for Dental Research 2018 \\ Reprints and permissions: \\ sagepub.com/journalsPermissions.nav \\ DOI: 10.1 I 77/00220345। 7743750 \\ journals.sagepub.com/home/adr
}

\author{
J.A. Horst
}

\begin{abstract}
Medical management of caries is a distinct treatment philosophy that employs topical minimally invasive therapies that treat the disease and is not merely prevention. This strategy is justified as an alternative or supplement to traditional care by significant disease recurrence rates following comprehensive operative treatment under general anesthesia. Silver diamine fluoride (SDF) is one agent to enable effective noninvasive treatment. The announcement of breakthrough therapy designation by the Food and Drug Administration (FDA) suggests that SDF may become the first FDA-approved drug for treating caries. Since our systematic review performed in April 20I5, 4 clinical trials have been completed, which inform an update to the application protocol and frequency regimen. Suggestions from these studies are to skip the rinsing step due to demonstration of safety in young children, start patients with high disease severity on an intensive regimen of multiple applications over the first few weeks, and continue with semiannual maintenance doses as previously suggested. Breakthroughs in elucidating the impact of SDF on the dental plaque microbiome inform potential opportunities for understanding caries arrest. SDF can be added to the set of evidence-based noninvasive methods to treat caries lesions in primary teeth, such as the Hall crown technique and sealing lesions with accessible margins.
\end{abstract}

Keywords: caries treatment, silver diamine fluoride, silver nitrate, evidence-based dentistry, topical anti-infective agents, tooth remineralization

Dental caries occurs when dental plaque bacteria ferment dietary sugars into acids that dissolve the tooth. Dental caries is the most prevalent human disease (Murray et al. 2012). More than $90 \%$ of adults in the United States have experienced caries (Dye et al. 2015). However, disparities in disease severity and access to care persist between high and low socioeconomic groups.

Treatment of the disease itself is needed: change the bacteria, strengthen the tooth, enhance the saliva, and decrease sugar consumption. Medical models of caries treatment attempt to accomplish these goals with antimicrobials, remineralizing agents, salivary stimulation, and dietary behavior modification. Yet there are no Food and Drug Administration (FDA)approved drugs for treating dental caries. Fluoridated toothpaste is approved by the FDA as an over-the-counter drug for preventing dental caries. High-concentration fluoride toothpaste and other fluoride products, including fluoride varnish and silver diamine fluoride, are cleared by the FDA as medical devices for treating tooth sensitivity.

\section{Disease Recurrence following Operative Treatment}

Operative approaches (e.g., fillings) are helpful to stop the progression of individual lesions. However, treatment should address the disease as well as existing signs of disease. The incidence of new caries lesions (disease recurrence) following comprehensive operative treatment reflects the success of treatments in stopping the disease process itself. Treatment of all lesions at once is commonly performed for children in the relatively ideal conditions of general anesthesia. Figure 1 summarizes the incidence of new caries lesions following treatment of cavities under general anesthesia (GA; adapted from Twetman and Dhar 2015). After $6 \mathrm{mo}, 38 \% \pm 1 \%$ of patients have new lesions (mean \pm standard deviation; Primosch et al. 2001; Chase et al. 2004; Berkowitz et al. 2011); this rises to $45 \% \pm 32 \%$ after $1 \mathrm{y}$ (Zhan et al. 2006; Hughes et al. 2012) and $62 \% \pm 15 \%$ after 2 y (Almeida et al. 2000; Foster et al. 2006; Amin et al. 2010). These relapse rates indicate a need for improvements in the care paradigm.

\section{Risk from Advanced Techniques}

Young children are increasingly sedated and anesthetized to enable operative treatment (e.g., fillings; Bruen et al. 2016). This approach poses a risk to life. Indeed, a Lexus-Nexus search found that the deaths of 44 children from sedation or general anesthesia to enable dental treatment were reported in the news media between 1980 and 2011 (Lee et al. 2013). Too many have shown up in the news since 2011. Yet there is no mandated public reporting, no mandated reporting from state dental boards to any federal agency, and no national database, so these reports underestimate the real incidence. A more comprehensive report from global data estimates a 1:327,684 risk of death from using general anesthesia for dental treatment

'Department of Biochemistry and Biophysics, University of California, San Francisco, CA, USA

\section{Corresponding Author:}

J.A. Horst, Department of Biochemistry and Biophysics, UCSF DeRisi Lab, 1700 4th St., QB3 Room 404, San Francisco, CA 94I58-2330, USA. Email: jahorst@gmail.com 


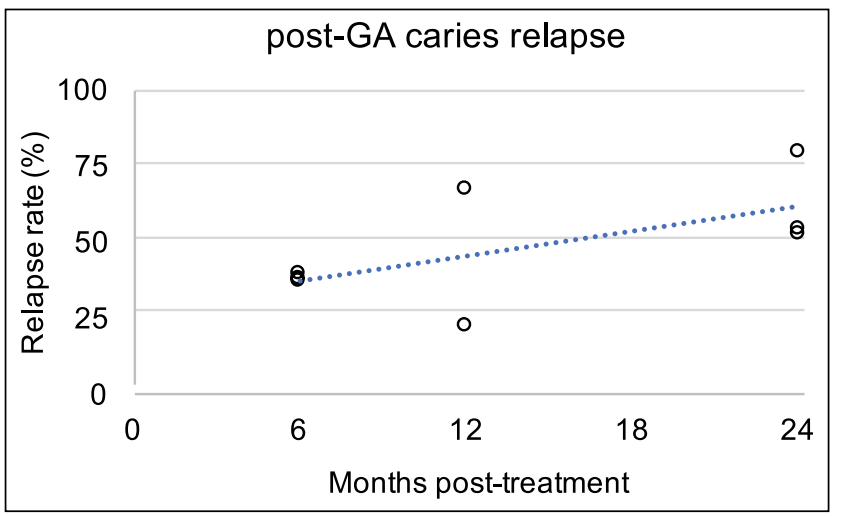

Figure I. Relapse of signs of dental caries following treatment under general anesthesia (GA). Incidence of new caries lesions following treatment under general anesthesia is plotted against time of evaluation. Linear regression follows $y=1.3 x+29.6$, with a correlation coefficient $R^{2}=0.4$. Adapted from Twetman and Dhar (20I5, Table 4). References: Almeida et al. 2000; Primosch et al. 200I; Chase et al. 2004; Foster et al. 2006; Zhan et al. 2006; Amin et al. 2010; Berkowitz et al. 20I I; Hughes et al. 2012 .

(Mortazavi et al. 2017). It is largely thought that in-office sedation by the operating dentist carries much higher risk, and it has been established that dental specialists carry the greatest risk of negative outcomes for sedation (Coté et al. 2000). Indeed, in a recent survey, over $75 \%$ of 439 responding dentists in Virginia said that at least one of their patients had experienced a sedationrelated emergency in their offices.

\section{Treatment to Achieve Prevention}

Silver diamine fluoride (SDF) is a brush-on liquid that stops $81 \%$ of dental caries lesions (Gao, Zhao, et al. 2016). This treatment success rate is similar to that of restorations placed under general anesthesia (Bücher et al. 2014): stopping lesion progress (caries arrest) appears to have the same effect on preventing pain from the lesion as restorations, but these approaches need to be compared directly in diverse clinical situations. In addition, lesion arrest is not the same as the incidence of new lesions (elaborated above for treatment under GA). In that vein, one of the most exciting aspects of SDF is the $58 \% \pm 22 \%$ decrease in new lesions after 1 to 3 y compared to no treatment or placebo controls, also outperforming all topical interventions except sealants (Chu et al. 2002; Llodra et al. 2005; Liu et al. 2012; Monse et al. 2012). The effective treatment of caries lesion sensitivity, albeit in the permanent teeth of adults (Castillo et al. 2011), further indicates SDF as an appropriate treatment for caries. SDF meets the goals of decreasing pain and incidence of new lesions.

\section{Stopping Caries Lesion Progression (Caries Arrest)}

Three clinical trials on caries arrest by SDF have been published since our systematic review (Fig. 2; Horst et al. 2016).
One trial in 3- to 4-y-old children documented a dose-response in both application frequency and concentration (Fung et al. 2016). Twice-annual application resulted in more arrested lesions after $18 \mathrm{mo}$; similarly, 38\% SDF (Saforide; Toyo Seiyaku Kasei Co. Ltd.) stopped more lesions than 12\% SDF (Cariostop; Biodinâmica Químicae Farmacêutica LTDA). This trend maintained after 24 and $30 \mathrm{mo}$, although the magnitude of effect for each regimen appeared to plateau at 18 mo (Fung et al. 2017). The higher effectiveness from increased frequency mimicked that shown previously (Zhi et al. 2012).

Another trial in 3- to 4-y-old children documented increased efficacy at 6 and 12 mo following intensive application (3 times in $2 \mathrm{wk}$ ), which was overcome in the single-application group by reapplication at 12 mo (Duangthip et al. 2016). These outcomes support both the concepts of intensive applications at the beginning of treatment and reapplying over longer periods of time. It should be noted that much lower arrest rates were seen in this study than others, which may be explained by the concentration of Cariostop actually having around one-third SDF instead of the advertised 30\% (Mei et al. 2013).

A trial in adults averaging $72 \mathrm{y}$ of age showed dramatically more effectiveness in arresting caries, 90\% (Li et al. 2016), than the $28 \%$ seen in the previous study of arrest in older adults (Zhang et al. 2013). This study also explored the application of potassium iodide (KI) after SDF to reduce discoloration, as the interaction of the 2 produces silver iodide that is yellowish white, instead of black from oxidized silver. This combination did not reduce effectiveness; on the contrary, there was a nonstatistically significant trend for higher effectiveness at all timepoints. It may be instructive to note that a similar trend in higher effectiveness at all timepoints was also observed following precipitation with tannic acid (Yee et al. 2009). Unfortunately, using KI did not make a significant change to the discoloration resulting from SDF treatment. Indeed, the intention of applying KI after SDF is to decrease color changes while remaining sealed and blocked from light, as under opaque glass ionomers (personal communication from the inventor, Graham Craig, 2017).

In total, 1,816 patients have been treated with SDF across 12 randomized clinical trials published in English. Pharmacokinetics (Vasquez et al. 2012) and gingival response (Castillo et al. 2011) have been assessed in adults. No significant harms have been noted. This would seem to indicate safety, but in reality, no prospective explicit measure of safety had been published in children. To address this question, we completed a doubleblind randomized placebo-controlled superiority trial of SDF in 66 children aged 3 to $5 \mathrm{y}$. We included a safety questionnaire to parents within $48 \mathrm{~h}$ of treatment and physical assessment at follow-up (Milgrom et al. 2017). This "Stopping Cavities" trial documented no adverse events within $21 \mathrm{~d}$ after application of blue-tinted SDF (Advantage Arrest; Elevate Oral Care LLC) without a rinse. Higher levels of arrest were observed in this trial $(72 \%)$, at $2 \mathrm{wk}$ versus the earliest trial outcome of $6 \mathrm{mo}$ (Fig. 2), which suggests that the effect dissipates with time. Concerns have been expressed about losing effectiveness by rinsing SDF away in the UCSF Protocol; the purpose was 


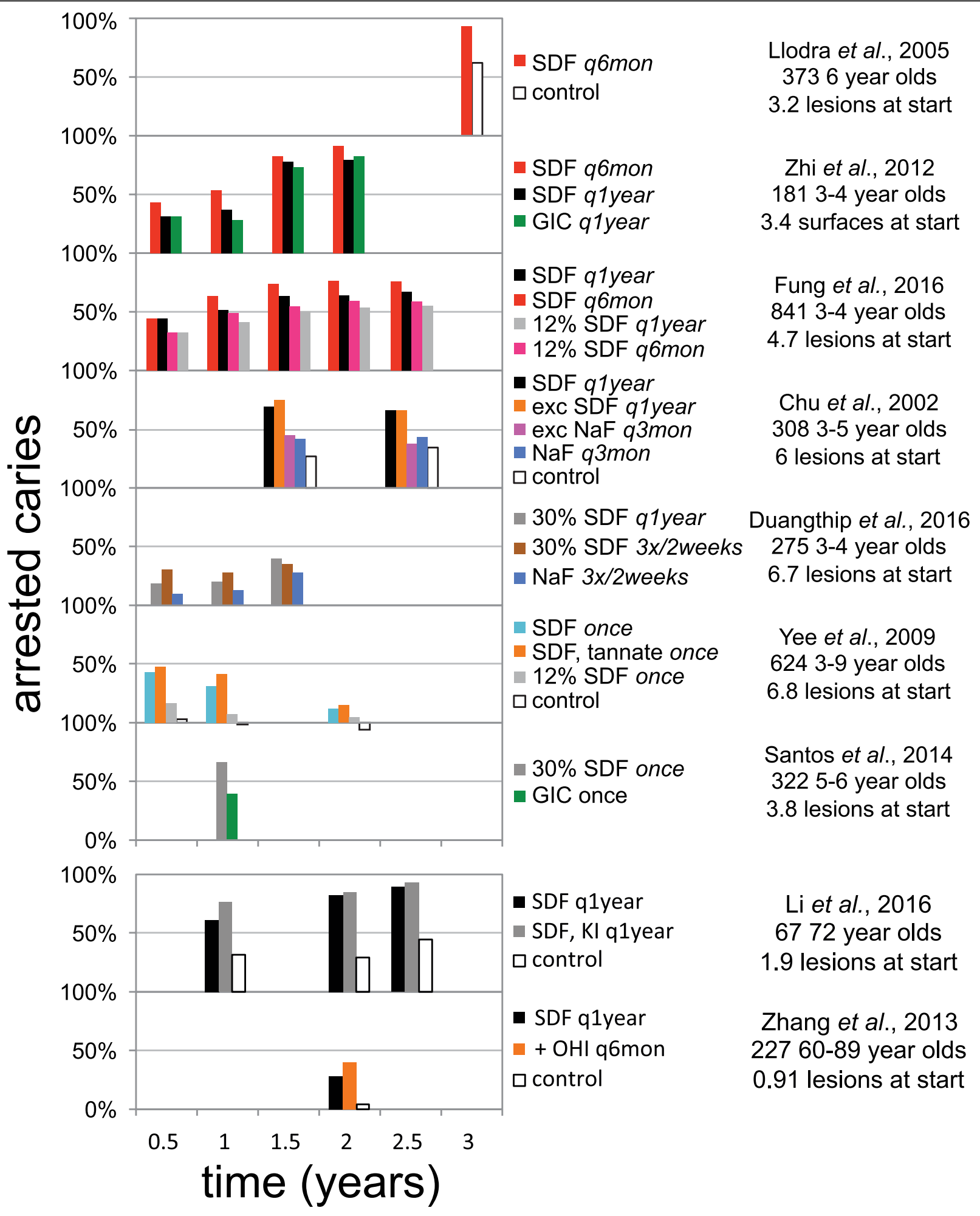

Figure 2. Graphic summary of randomized controlled trials demonstrating caries arrest after topical treatment with silver diamine fluoride (SDF). Studies are arranged vertically by frequency of SDF application. Caries arrest is defined as the fraction of initially active carious lesions that became inactive and firm to a dental explorer. SDF (38\% unless noted otherwise); GIC, glass ionomer cement; NaF, $5 \%$ sodium fluoride varnish; + OHI q6mon, SDF every year and oral hygiene instructions every 6 mo; qlyear, every year; q3mon, every 3 mo; q6mon, every 6 mo. Updated from Horst et al. (2016). 
concern of safety without it (Horst et al. 2016). The lack of adverse events observed in this study leaves no apparent reason to continue rinsing lesions after SDF treatment. It is often appropriate to rinse or wipe the tongue only, to remove the taste after SDF application, or to cover the taste by giving the child something with a strong desirable flavor.

From these 4 trials, clinicians may also consider intensive application regimens (e.g., 3 times in $2 \mathrm{wk}$ ) and then spreading out further applications over time, skipping the rinse, and further reassurance of a dose-response by application frequency, the need for repeated application over time, and a range from $28 \%$ to $90 \%$ arrest in treating root caries in older adults.

A recent systematic review found various comparative clinical studies and case series published in Chinese, Japanese, Portuguese, and Spanish (Gao, Zhao, et al. 2016). After excluding studies by quality and risks of bias, they estimated an $81 \%$ likelihood of caries arrest in primary teeth $(95 \%$ confidence interval, 68\%-89\%) following treatment with $38 \%$ SDF regardless of application regimen and duration of evaluation. A recent case series in Oregon showed 100\% arrest after 3 mo (Clemens et al. 2017).

Five clinical trials compare caries arrest following treatment with SDF against a control or placebo. In 2 of the studies, the placebo group showed no significant pattern of caries arrest from baseline (Yee et al. 2009; Tan et al. 2010). However, 3 of the studies showed a significant effect, ranging from $34 \%$ to $62 \%$ of lesions becoming arrested (Chu et al. 2002; Llodra et al. 2005; Li et al. 2016). Thus, it is probable that some lesions do not need treatment and will become arrested without intervention. Consequently, the $81 \%$ of caries lesions estimated to arrest following SDF treatment probably include some that would arrest without SDF treatment.

\section{Other Noninvasive Approaches to Arrest Caries}

While some medicaments decrease the incidence of new lesions, almost no noninvasive therapies available in the United States have been shown to stop caries lesions in the dentin. Fluoride varnish reverses two-thirds of enamel lesions (Gao, Zhang, et al. 2016) but makes no impact on dentin caries compared to placebo (Chu et al. 2002). While clinical studies during the early and midpart of the past century showed highly inconsistent outcomes from silver nitrate, use to treat dentin caries in the early 1800 s and 1900 s was common enough to suggest that there is some effect (Black 1908). Sealing in caries, where circumferential enamel is accessible, seems to be the only effective noninvasive alternative (Mertz-Fairhurst et al. 1998).

The Hall crown technique similarly achieves the goal of sealing in caries lesions without removal of any carious material, although the crown margins dive into the gingival sulcus and thus might be considered to have some amount of invasiveness. Nonetheless, the Hall crown does not require accessible cavity margins or removal of any tooth structure. Moreover, clinical outcomes of the Hall technique show superiority to traditional restorations in both comparative clinical trials (Innes et al. 2011; Santamaria et al. 2014).
SDF is the combination of an antimicrobial (Ag, 25\% w/v), a remineralizing agent $(\mathrm{F}, 5 \%)$, and a stabilizing agent that happens to also be an antiseptic (ammonia, 8\%). As mentioned above, none of the components of SDF have been shown to be consistently effective in treating dentinal caries lesions on their own. This suggests that future gains may be made by further or different combinations.

\section{Regulatory Progress}

In 2014, the FDA cleared SDF as a medical device for treating tooth sensitivity. In 2016, the FDA awarded breakthrough therapy status as a commitment to an application for approval of SDF as a drug to treat severe early childhood caries (press release from Elevate Oral Care, October 30, 2016). Breakthrough therapy status does not mean approval; rather, it is a commitment to evaluate and assist in the related new drug application, for a life-threatening disease with no available treatment. Nonetheless, this and the consistent response in many previous clinical trials suggest that SDF will be the first FDA drug to treat dental caries. Canada recently approved SDF with an indication of "anti-caries" (press release from Oral Sciences, March 8, 2017). The Indian Health Service released a policy supporting the use of silver ion antimicrobials (SDF or the combination of silver nitrate and fluoride varnish) in their clinics. The American Dental Association Council on the Advancement of Access and Prevention has written a resolution in support of use of SDF for caries. The American Academy of Pediatric Dentistry has adopted a policy and guideline supporting use to treat caries as well. This wave of support and interest is appropriate given the many large clinical trials that demonstrate effectiveness.

\section{SDF Adoption}

Recent conference presentations described studies that document high levels of acceptance of the stains caused by SDF. An elegant study in New York City asked 33 parents to choose between treatment with SDF or white plastic resin fillings, informing them of the considerations to enable these treatments (Tesoriero and Lee 2016). All parents of "uncooperative" children chose SDF, while two-thirds of parents of other children also chose SDF. A sex disparity emerged, wherein $86 \%$ of parents chose SDF for their sons, while only $61 \%$ chose SDF for their daughters; still, the majority prefer a black stain and uncertainty about outcome over an injection, drill, and prolonged treatment time. The implication is that parents would rather their children have blemishes than experience pain.

Another similar study nearby asked about hypothetical acceptability of the stain. While only $32 \%$ of parents accepted the idea of SDF for treating anterior teeth initially, a potential requirement of general anesthesia to enable operative treatment drove acceptance up to $70 \%$ (Crystal et al. 2017). It is interesting to consider how responses might have differed if the studies were conducted after the December 2016 FDA Black Box Warning on the use of GA in pregnancy and before the third birthday. Meanwhile, most pediatric dentistry residencies (Nelson et al. 2016) and half of dental school programs are teaching trainees about SDF (Ngoc et al. 2017). 
A recent study evaluated the perception of parents whose children were treated with SDF in the case series in Oregon mentioned previously. Most parents strongly agreed that "SDF application is an easy process; I am comfortable with discoloration of cavities after SDF placement; SDF application was pain free for my child; The taste of SDF was acceptable to my child," and all the remaining parents responded as either agreeing or being neutral, except 1 who disagreed about comfort with discoloration (Clemens et al. 2017). Indeed, the first clinical trial of SDF published in English found that parental satisfaction with their children's dental appearance was not different between baseline and $2 \mathrm{y}$ later or between treatment groups. This study in Guangzhou, China, found that $7 \%$ of parents described dark teeth as the reason for dissatisfaction, with the remainder concerned about signs of decay in the anterior teeth generally (Chu et al. 2002). This suggests a very high acceptance rate of SDF in cultures as disparate as Guangzhou and Oregon.

\section{SDF Microbial Mechanisms}

While considerable in vitro experiments have documented that SDF inactivates every tested protein and bacterium, until the Stopping Cavities trial, no clinical microbiology had been published. The question arose: if SDF kills all bacteria, which microbes are present in the nutrient-rich environment of the SDF-treated caries lesion? To address this question, we performed massively parallel RNA sequencing of a pilot set of plaque samples in the Stopping Cavities trial, taken from 2 caries lesions before and $2 \mathrm{wk}$ after placebo or control treatments for each child (Milgrom et al. 2017). RNA was used as a proxy for vitality, to enable measurement of all vital microbes; RNA degrades within an hour of production in these conditions. Care was taken to minimize inflow of saliva. The hypothesis was that the relative abundance of caries-associated bacteria would be reduced in the treatment group, but surprisingly, no such changes were observed. Mild increases were seen for only a few bacteria not related to caries and that pose no known threat. A trend toward increased diversity was seen, rather than the expected decrease that is ubiquitously observed following a course of systemic antibiotics. This signals safety. Abundant high-quality RNA was retrieved, which was also surprising. The RNA sequences were also scoured for antibiotic or antimetal resistance genes, and these were not changed by treatment. While this was a pilot study in a subset of patients, it is impressive that the microbial composition of the dental plaque on the surface of treated lesions did not significantly change.

\section{Summary}

The appropriateness of traditional operative dentistry under sedation and general anesthesia as the first line of treatment for dental caries in primary teeth is in question. The FDA Black Box Warning against general anesthetics in young children urges a paradigm shift. Clearance of SDF in the United States provides an agent for change to noninvasive caries management. Rapid adoption despite the nonesthetic results indicates preference against the discomfort required by traditional operative dentistry, which is further supported by surveys and parent choices. New clinical trial data suggest starting with more frequent applications and decreasing frequency with time, while maintaining at least annual application and removing the rinse step. Our recent work documents a surprising lack of changes to the dental plaque microbiota following SDF treatment. While more work needs to be done to understand and anticipate treatment failure, all new data support the effectiveness and safety for treatment of dental caries by SDF.

\section{Author Contributions}

J.A. Horst, contributed to conception, design, data acquisition, analysis, and interpretation, drafted and critically revised the manuscript. The author gave final approval and agrees to be accountable for all aspects of the work.

\section{Acknowledgments}

This work was supported by National Institutes of Health National Institute of Dental and Craniofacial Research grant T32-DE007306. Thanks to Drs. Jason Hirsch, John Frachella, Steve Duffin, Jeanette MacLean, Jong Seto, and Peter Milgrom for thoughtful discussions on the clinical use of SDF for treating dental caries. Thanks to Dr. John Featherstone for guidance on forming the manuscript. The author's salary is now paid by UCSF from a gift from Advantage Silver Dental Arrest. The author has collaborated with and taught continuing education courses for the owners of Advantage Silver Dental Arrest. The author declares no other potential conflicts of interest with respect to the authorship and/or publication of this article.

\section{References}

Almeida AG, Roseman MM, Sheff M, Huntington N, Hughes CV. 2000. Future caries susceptibility in children with early childhood caries following treatment under general anesthesia. Pediatr Dent. 22(4):302-306.

Amin MS, Bedard D, Gamble J. 2010. Early childhood caries: recurrence after comprehensive dental treatment under general anaesthesia. Eur Arch Paediatr Dent. 11(6):269-273.

Berkowitz RJ, Amante A, Kopycka-Kedzierawski DT, Billings RJ, Feng C. 2011. Dental caries recurrence following clinical treatment for severe early childhood caries. Pediatr Dent. 33(7):510-514.

Black GV. 1908. The pathology of the hard tissues of the teeth. Chicago (IL): Medico-Dental Publishing Company.

Bruen BK, Steinmetz E, Bysshe T, Glassman P, Ku L. 2016. Potentially preventable dental care in operating rooms for children enrolled in Medicaid. J Am Dent Assoc. 147(9):702-708.

Bücher K, Tautz A, Hickel R, Kühnisch J. 2014. Longevity of composite restorations in patients with early childhood caries (ECC). Clin Oral Investig. 18(3):775-782.

Castillo JL, Rivera S, Aparicio T, Lazo R, Aw TC, Mancl LL, Milgrom P. 2011. The short-term effects of diammine silver fluoride on tooth sensitivity: a randomized controlled trial. J Dent Res. 90(2):203-208.

Chase I, Berkowitz RJ, Proskin HM, Weinstein P, Billings R. 2004. Clinical outcomes for early childhood caries (ECC): the influence of health locus of control. Eur J Paediatr Dent. 5(2):76-80.

Chu CH, Lo ECM, Lin HC. 2002. Effectiveness of silver diamine fluoride and sodium fluoride varnish in arresting dentin caries in Chinese pre-school children. J Dent Res. 81(11):767-770.

Clemens J, Gold J, Chaffin J. 2017. Effect and acceptance of silver diamine fluoride treatment on dental caries in primary teeth. J Public Health Dent [epub ahead of print 27 Jul 2017] in press. doi: 10.1111/jphd.12241.

Coté CJ, Karl HW, Notterman DA, Weinberg JA, McCloskey C. 2000. Adverse sedation events in pediatrics: analysis of medications used for sedation. Pediatrics. 106(4):633-644. 
Crystal YO, Janal MN, Hamilton DS, Niederman R. 2017. Parental perceptions and acceptance of silver diamine fluoride staining. J Am Dent Assoc. 148(7):510-518.e4

Duangthip D, Chu CH, Lo ECM. 2016. A randomized clinical trial on arresting dentine caries in preschool children by topical fluorides -18 month results. J Dent. 44:57-63.

Dye B, Thornton-Evans G, Li X, Iafolla T. 2015. Dental caries and tooth loss in adults in the United States, 2011-2012. NCHS Data Brief. (197): 197.

Foster T, Perinpanayagam H, Pfaffenbach A, Certo M. 2006. Recurrence of early childhood caries after comprehensive treatment with general anesthesia and follow-up. J Dent Child (Chic). 73(1):25-30.

Fung MH, Duangthip D, Wong MC, Lo EC, Chu CH. 2016. Arresting dentine caries with different concentration and periodicity of silver diamine fluoride. JDR Clin Trans Res. 1(2):143-152.

Fung MH, Duangthip D, Wong MC, Lo EC, Chu CH. 2017. Randomized clinical trial of $12 \%$ and $38 \%$ silver diamine fluoride treatment. J Dent Res [epub ahead of print 28 Aug 2017] in press. doi:10.1177/0022034517728496

Gao SS, Zhang S, Mei ML, Lo EC, Chu CH. 2016. Caries remineralisation and arresting effect in children by professionally applied fluoride treatment - a systematic review. BMC Oral Health. 16:12.

Gao SS, Zhao IS, Hiraishi N, Duangthip D, Mei ML, Lo ECM, Chu CH. 2016. Clinical trials of silver diamine fluoride in arresting caries among children. JDR Clin Trans Res. 1(3):201-210.

Horst JA, Ellenikiotis H, Milgrom PL. 2016. UCSF protocol for caries arrest using silver diamine fluoride: rationale, indications and consent. J Calif Dent Assoc. 44(1):16-28.

Hughes CV, Dahlan M, Papadopolou E, Loo CY, Pradhan NS, Lu SC, Mathney JMJ, Bravoco A, Kent RL, Tanner ACR. 2012. Aciduric microbiota and mutans streptococci in severe and recurrent severe early childhood caries. Pediatr Dent. 34(2):e16-e23.

Innes NP, Evans DJ, Stirrups DR. 2011. Sealing caries in primary molars: randomized control trial, 5-year results. J Dent Res. 90(12):1405-1410.

Lee HH, Milgrom P, Starks H, Burke W. 2013. Trends in death associated with pediatric dental sedation and general anesthesia. Paediatr Anaesth. 23(8):741-746.

Li R, Lo EC, Liu BY, Wong MC, Chu CH. 2016. Randomized clinical trial on arresting dental root caries through silver diammine fluoride applications in community-dwelling elders. J Dent. 51:15-20.

Liu BY, Lo EC, Chu CH, Lin HC. 2012. Randomized trial on fluorides and sealants for fissure caries prevention. J Dent Res. 91(8):753-758.

Llodra JC, Rodriguez A, Ferrer B, Menardia V, Ramos T, Morato M. 2005. Efficacy of silver diamine fluoride for caries reduction in primary teeth and first permanent molars of schoolchildren: 36-month clinical trial. J Dent Res. 84(8):721-724.

Mei ML, Chu CH, Lo EC, Samaranayake LP. 2013. Fluoride and silver concentrations of silver diammine fluoride solutions for dental use. Int J Paediatr Dent. 23(4):279-285.

Mertz-Fairhurst EJ, Curtis JW, Ergle JW, Rueggeberg FA, Adair SM. 1998 Ultraconservative and cariostatic sealed restorations: results at year 10 . J Am Dent Assoc. 129(1):55-66.
Milgrom P, Horst JA, Ludwig S, Rothen M, Chaffee BW, Lyalina S, Pollard KS, DeRisi JL, Mancl L. 2017. Topical silver diamine fluoride for dental caries arrest in preschool children: a randomized controlled trial and microbiological analysis of caries associated microbes and resistance gene expression. J Dent. S0300-5712(17):30212-30219.

Monse B, Heinrich-Weltzien R, Mulder J, Holmgren C, van Palenstein Helderman WH. 2012. Caries preventive efficacy of silver diammine fluoride (SDF) and ART sealants in a school-based daily fluoride toothbrushing program in the Philippines. BMC Oral Health. 12:52.

Mortazavi H, Baharvand M, Safi Y. 2017. Death rate of dental anaesthesia. J Clin Diagn Res. 11(6):ZE07-ZE09.

Murray CJ, Vos T, Lozano R, Naghavi M, Flaxman AD, Michaud C, Ezzati M, Shibuya K, Salomon JA, Abdalla S, et al. 2012. Disability-adjusted life years (DALYs) for 291 diseases and injuries in 21 regions, 1990-2010: a systematic analysis for the Global Burden of Disease Study 2010. Lancet. 380(9859):2197-2223.

Nelson T, Scott JM, Crystal YO, Berg JH, Milgrom P. 2016. Silver diamine fluoride in pediatric dentistry training programs: survey of graduate program directors. Pediatr Dent. 38(3):212-217.

Ngoc CN, Ahmed S, Mehta R, Donovan T, Zandona AF. 2017. Silver diamine fluoride in U.S. dental schools curriculum. Poster session presented at: International Association of Dental Research 95th General Session; San Francisco, CA.

Primosch RE, Balsewich CM, Thomas CW. 2001. Outcomes assessment an intervention strategy to improve parental compliance to follow-up evaluations after treatment of early childhood caries using general anesthesia in a Medicaid population. ASDC J Dent Child. 68(2):102-8, 180.

Santamaria RM, Innes NP, Machiulskiene V, Evans DJ, Splieth CH. 2014. Caries management strategies for primary molars: 1-yr randomized control trial results. J Dent Res. 93(11):1062-1069.

Tan HP, Lo EC, Dyson JE, Luo Y, Corbet EF. 2010. A randomized trial on root caries prevention in elders. J Dent Res. 89(10):1086-1090.

Tesoriero J, Lee A. 2016. Parental acceptance of silver diamine fluoride. Poster session presented at: American Association of Pediatric Dentistry 69th Annual Session; San Antonio, TX.

Twetman S, Dhar V. 2015. Evidence of effectiveness of current therapies to prevent and treat early childhood caries. Pediatr Dent. 37(3):246-253.

Vasquez E, Zegarra G, Chirinos E, Castillo JL, Taves DR, Watson GE, Dills R, Mancl LL, Milgrom P. 2012. Short term serum pharmacokinetics of diammine silver fluoride after oral application. BMC Oral Health. 12:60.

Yee R, Holmgren C, Mulder J, Lama D, Walker D, van Palenstein Helderman W. 2009. Efficacy of silver diamine fluoride for arresting caries treatment. J Dent Res. 88(7):644-647.

Zhan L, Featherstone JDB, Gansky SA, Hoover CI, Fujino T, Berkowitz RJ, Besten Den PK. 2006. Antibacterial treatment needed for severe early childhood caries. J Public Health Dent. 66(3):174-179.

Zhang W, McGrath C, Lo ECM, Li JY. 2013. Silver diamine fluoride and education to prevent and arrest root caries among community-dwelling elders. Caries Res. 47(4):284-290.

Zhi QH, Lo EC, Lin HC. 2012. Randomized clinical trial on effectiveness of silver diamine fluoride and glass ionomer in arresting dentine caries in preschool children. J Dent. 40(11):962-967. 